\title{
Tumor budding and laminin $5-\gamma 2$ in squamous cell carcinoma of the external auditory canal are associated with shorter survival
}

\author{
Yasuko Okado ${ }^{1,2}$, Mikiko Aoki ${ }^{1}$, Makoto Hamasaki ${ }^{1}$, Kaori Koga' ${ }^{1}$,Takayuki Sueta², Hideki Shiratsuchii,4, \\ Yoshinao Oda ${ }^{5}$, Takashi Nakagawa ${ }^{2}$ and Kazuki Nabeshima ${ }^{1 *}$
}

\begin{abstract}
Squamous cell carcinoma (SCC) of the external auditory canal (EAC) is rare, usually presents at an advanced stage, and is a more aggressive tumor with poor prognosis. The University of Pittsburgh TNM staging system commonly used in prognostication is not perfect, and more accurate biomarkers predicting prognosis are needed. Tumor budding is an established negative prognostic factor at the invasive front in colorectal cancer. Moreover, immunohistochemical studies showed that laminin 5- $\gamma 2(\operatorname{Ln} 5-\gamma 2)$ is expressed at the invasive front in tumor or tumor budding cells. We assessed the prognostic significance of tumor budding and Ln5- $\gamma 2$ expression by performing Ln5- $\gamma 2$ immunohistochemistry and evaluated the degree of tumor budding in pre-treatment biopsy specimens, and investigated their correlations to clinicopathological parameters in patients with SCC of the EAC. Patients whose tumors had high budding grade and Ln5- $\gamma 2$ expression had significantly shorter survival times. Budding grade was significantly correlated with Ln5- $\gamma 2$ expression. Multivariate analysis revealed that high budding grade predicted poorer prognosis regardless of disease stage. Our results suggested that budding grade and Ln5- $\gamma 2$ expression can be used as indicators of poor prognosis in patients with SCC of the EAC.
\end{abstract}

Keywords: Squamous cell carcinoma, External auditory canal, Tumor budding, Laminin 5- $\gamma 2$

\section{Background}

Squamous cell carcinoma (SCC) of the external auditory canal (EAC) is an aggressive malignancy with a poor prognosis. The reported incidence is less than six cases per million per year, accounting for $0.2 \%$ of all tumors of the head and neck, with a reported 5-year disease-specific survival ranging from 19 to $48 \%$ (Moffat et al. 2000; Bacciu et al. 2013; Prasad et al. 2014; Masterson et al. 2014). Because of the rarity of this tumor, it has been difficult for any single institution to analyze the clinical data and formulate an optimal evaluation and treatment strategy. Another impediment to the study of SCC of the

\footnotetext{
*Correspondence: kaznabes@fukuoka-u.ac.jp

${ }^{1}$ Department of Pathology, Fukuoka University School of Medicine and Hospital, 7-45-1 Nanakuma, Jonan-ku, Fukuoka 814-0180, Japan Full list of author information is available at the end of the article
}

EAC is the lack of a universally accepted staging system (Bacciu et al. 2013; Prasad et al. 2014; Masterson et al. 2014; Nakagawa et al. 2006). Currently, there is no recognized stand-alone American Joint Committee on Cancer (Chicago, IL, USA) or International Union Against Cancer (Geneva, Switzerland) staging system for SCC of the EAC (Prasad et al. 2014). A revised University of Pittsburgh (Pittsburg, PA, USA) staging system was proposed in the year 2000 (Moody et al. 2000). This system is the classification tool that is commonly used for prognosis and treatment decisions in patients with SCC of the EAC (Bacciu et al. 2013; Prasad et al. 2014; Masterson et al. 2014; Nakagawa et al. 2006; Moody et al. 2000). However, this tool does not correctly classify every patient (Prasad et al. 2014; Mazzoni et al. 2014); although the number is small, there are some patients with tumors that recur

\section{至 Springer}

(C) 2015 Okado et al. This article is distributed under the terms of the Creative Commons Attribution 4.0 International License (http://creativecommons.org/licenses/by/4.0/), which permits unrestricted use, distribution, and reproduction in any medium, provided you give appropriate credit to the original author(s) and the source, provide a link to the Creative Commons license, and indicate if changes were made. 
locally despite their categorization as low-risk and, conversely, patients with longer survival times despite their categorization as high-risk, based on the TNM system (Prasad et al. 2014). The failure of TNM staging to serve as a reliable prognostic system for patients with intermediate-stage tumors may be overcome by considering morphologic, molecular, or treatment-related factors to stratify patients more precisely into different risk categories. Thus, search for biomarkers that reflect the biologic characteristics of SCC of the EAC and treatment outcome prediction is crucial (Marioni et al. 2012, 2013).

Tumor budding/sprouting is a histological feature at the invasive front of a tumor and an established negative prognostic factor when present in resected colorectal cancer and preoperative rectal cancer biopsy specimens (Morodomi et al. 1989; Hase et al. 1993; Ueno et al. 2002; Zlobec and Lugli 2010; Mitrovic et al. 2012; Rogers et al. 2014; Satoh et al. 2014). The concept of tumor budding/ sprouting was first described in the 1950s by Imai, who postulated that the presence of "sprouting" at the invasive edge of a carcinoma indicates a more rapid tumor growth rate (Imai 1960). Tumor budding is thought to represent an active invasive form of cancer; specifically, it is the isolation and mobilization of cancer cells from a main tumor mass during the early stages of tumor invasion (Hase et al. 1993). Tumor cells that have undergone the epithelial-to-mesenchymal transition (EMT) are indicated histologically by the presence of tumor budding (Mitrovic et al. 2012). Tumor budding is predictive of lymph node metastasis, vascular and lymphatic invasion, non-responsiveness to neoadjuvant chemo-radiotherapy, distant metastasis, local recurrence, and a poor prognosis (Morodomi et al. 1989; Ueno et al. 2002; Koike et al. 2008; Brown et al. 2010; Sarioglu et al. 2010; Zlobec and Lugli 2010; Luo et al. 2012; Mitrovic et al. 2012; Rogers et al. 2014; Satoh et al. 2014). A high level of tumor budding/sprouting is associated with a poorer prognosis in several human solid cancers, including some types of SCC, e.g., esophageal SCC (Koike et al. 2008; Brown et al. 2010), laryngeal carcinoma (Sarioglu et al. 2010), and nasopharyngeal carcinoma (Luo et al. 2012). For head and neck SCC, the importance of invasion pattern for prognosis has been extensively addressed since seventies (Willen et al. 1975; Anneroth et al. 1987; Bryne et al. 1992, 1998). The studies have shown that it is not the differentiation grade but the invasion pattern that is important for assessment of clinicopathological aggressiveness. Particularly, the invasion pattern classified as Grade or Score 4, which referred to neoplasms with a marked diffuse, widespread cellular invasion of the neoplasm in single neoplastic cells or in small groups of cells, corresponds to tumor budding mentioned herein (Anneroth et al. 1987). More recently, a non-cohesive invasive front is reported to be an indicator of metastasis in the SCC of the ear auricle, which is known to metastasize to the regional lymph nodes more frequently than SCC at other sites (Clark et al. 2010).

Previously, our laboratory showed that laminin 5- $\gamma 2$ (Ln5- $\gamma 2$ ) has a role in invasion of cutaneous SCC (Hamasaki et al. 2011). We also reported that even in the early stages of SCC of the EAC, diffuse expression of Ln5- $\gamma 2$ is associated with a poor prognosis (Nakagawa et al. 2013). Laminin 5, a heterotrimer composed of three different Laminin chains ( $\alpha 3-, \beta 3-, \gamma 2-)$, is the major component of the basement membrane in most adult tissues (Nguyen et al. 2000). One of the chains, Ln5- $\gamma 2$, is a specific marker for invasive tumors because it is frequently expressed as a monomer in several types of malignant tumors (Nguyen et al. 2000; Koshikawa et al. 2008). Several immunohistochemical studies have found that Ln5$\gamma 2$ expression is observed exclusively in the cytoplasm of budding cells at the invasive front in many types of human cancers, such as colon, and breast cancer, and SCC of the esophagus, cervix, and skin (Pyke et al. 1995; Yamamoto et al. 2001; Hamasaki et al. 2011).

To our knowledge, the degree of tumor budding in SCC of the EAC has not yet been described. The aim of this study was to assess the degree of tumor budding and its correlation to Ln5- $\gamma 2$. We also performed an immunohistochemical analysis of Ln5- $\gamma 2$ in pre-treatment ear cancer biopsy specimens to investigate the prognostic significance of $\operatorname{Ln} 5-\gamma 2$ expression.

\section{Methods \\ Patients}

We retrospectively reviewed clinicopathological data for 46 patients with primary SCC of the EAC, for whom the pretreatment tissue specimens were available, and who were treated by the same strategy at the Department of Otorhinolaryngology, Kyushu University Hospital (Fukuoka, Japan) from January 1998 to March 2006 and at the Department of Otorhinolaryngology, Fukuoka University Hospital (Fukuoka, Japan) from April 2006 to December 2014. Patients who underwent chemotherapy or radiation prior to biopsy, patients for whom specimens were not available, or patients who failed to follow our treatment plan, were excluded from the study. As long as the provisions for maintaining patient anonymity are followed, tissues from biopsy samples can be used for research purposes per a standard treatment agreement with Kyushu University and Fukuoka University Hospitals. The study protocol was approved by the Institutional Review Board (The Ethics Committee) of Kyushu University (No. 26-185) and Fukuoka University (No. 12-7-13) Hospitals. Clinical stage was determined using the staging protocol proposed by the University of Pittsburgh (Moody et al. 2000). Before treatment, the 
extent of disease was estimated in all patients by physical examination and imaging studies [Computed tomography $(\mathrm{CT})$, and magnetic resonance imaging (MRI)]. The invasion of the canal wall of the internal carotid artery, jugular bulb, otic capsule, and dura mater was determined by CT, and the extension to the soft tissue, petrous apex, middle ear, mastoid, temporomandibular joint, and parotid gland was distinguished by MRI. CT and MRI scans were routinely conducted every 6 months, for 3 years, after therapy. After 3 years, CT and MRI were performed annually. The surgical or post-irradiation scarring was often indistinguishable from residual or recurrent disease. An absence of any signs of enlargement of a space-occupying lesion, was designated as "no evidence of disease". The follow-up period for the complete series ranged from 4 to 66 months (median, 34 months).

\section{Tissue samples and immunohistochemistry (IHC)}

Biopsy specimens were fixed in $10 \%$ formalin and processed into paraffin blocks. Paraffinized tissue blocks were sectioned (4- $\mu \mathrm{m}$ thickness), deparaffinized, and hydrated in descending alcohol dilutions. For anticytokeratin (CK) antibody staining, sections were immersed in $3 \%$ hydrogen peroxide in water for $10 \mathrm{~min}$ at room temperature (RT) to block endogenous peroxidase activity, and heated in $10 \mathrm{mM}$ ethylenediaminetetraacetic acid (EDTA) buffer ( $\mathrm{pH}$ 8.0) in a microwave $(700 \mathrm{~W})$ for $10 \mathrm{~min}$ to retrieve epitopes before staining. For anti-Ln5- $\gamma 2$ antibody staining, sections were immersed in $0.05 \%$ protease XXIV (Sigma-Aldrich, Tokyo, Japan) for 15 min at RT to block endogenous peroxidase activity. The sections were incubated with anti-human CK monoclonal antibody (AE1/AE3, Dako; 1:200), or anti-human Ln5- $\gamma 2$ monoclonal antibody (D4B5, Temecula, CA, USA; 1:200), for $1 \mathrm{~h}$ at RT (for $\mathrm{CK}$ ), or for $1 \mathrm{~h}$ at $37{ }^{\circ} \mathrm{C}$ (for Ln5- $\gamma 2$ ). The sections were then washed in Tris-buffered saline (TBS) and incubated for $30 \mathrm{~min}$ at RT with EnVision reagent conjugated horseradish peroxidase (Dako; for CK), or Histofine reagent conjugated to alkaline phosphatase (Nichirei Bioscience; for Ln5- $\gamma 2$ ). Immunoreactive proteins were visualized with 3,3'-diamino-benzidine (DAB; Dako; for $\mathrm{CK}$ ), or New Fuchsin substrate kit (Nichirei Bioscience, Tokyo, Japan; for Ln5- $\gamma 2$ ), followed by counterstaining with Mayer's hematoxylin (for CK), or methylgreen (for Ln5- $\gamma 2$ ). The stained sections were evaluated semiquantitatively by two independent observers who were blinded to the clinical data.

\section{Grading for tumor budding/sprouting}

Based on the Japanese Classification of Colorectal Carcinoma (JCCC) (Rectum JSfCotCa 2013), we defined tumor budding/sprouting as a cancer cell nest consisting of five or fewer cells that infiltrated the interstitium at the invasive margin of the cancer. After selecting an area in which budding/sprouting was most intensive, the buds were counted in a field measuring $0.785 \mathrm{~mm}^{2}$ through a $20 \times$ objective lens (WHK $10 \times$ ocular lens; Olympus, Tokyo, Japan). Depending on the number of buds, we used the modified grading system proposed by Satoh et al. (2014) for estimation of tumor budding: Grade 0, no budding (Fig. 1a, e); Grade 1, 1-4 buds (Fig. 1b, f); Grade 2, 5-9 buds (Fig. 1c, g); Grade 3, $\geq 10$ buds (Fig. 1d, h). We used this grading system to evaluate tumor budding in H\&E- (Fig. 1a-d) and CKimmunostained (Fig. 1e-h) sections. The degree of tumor budding was classified as low grade or high grade, corresponding to $0-9$ (grades 0,1 , and 2) or $\geq 10$ budding foci (grade 3) in one field, respectively, according to Ueno et al. (2002). This grading system has been proven to be have prognostic significance, is easily applied, and is frequently used in tumor budding studies (Mitrovic et al. 2012).

\section{Immunohistochemical analysis of Ln5- ${ }^{2}$}

Immunoreactivity for Ln5- $\gamma 2$ was observed as cytoplasmic staining in carcinoma cells. The immunostaining result was considered to be negative if $<10 \%$ of the tumor cells were stained. In specimens considered to have a positive result, the tumor staining pattern was assessed as marginal (Fig. 2a) or diffuse (Fig. 2b), and was quantitated on a scale from 1 to 4 based on the percentage of positively-stained tumor cells. The scale was as follows: $1+, 10-24 \%$ positive (Fig. 2 c); $2+, 25-49 \%$ positive (Fig. 2d); 3+, 50-74 \% positive (Fig. 2e); and 4+, $75-100 \%$ positive (Fig. 2f). Each tumor was then classified as low-expression (1+ and 2+) or high-expression $(3+$ and $4+)$.

\section{Statistical analysis}

The relationships between several clinicopathological parameters and the results of budding assessments and IHC were evaluated using the Student's- $t$ test for continuous variables, and Fisher's exact test for categorical variables. Disease-specific survival curves were plotted by the Kaplan-Meier method, and $P$ values were calculated using the log-rank test. A univariate analysis was performed for each clinicopathological parameter. Multivariate analysis and the Cox proportional-hazard model were used to determine the independent prognostic factors. A $P$ value $<0.05$ was considered statistically significant. All data analyses were conducted with JMP10.0.2 (SAS Institute Inc., Cary, NC, USA) for Windows.

\section{Results}

\section{Clinical and histopathological findings}

Table 1 summarizes the clinicopathological characteristics of 46 patients [25 male and 21 female; age range, 

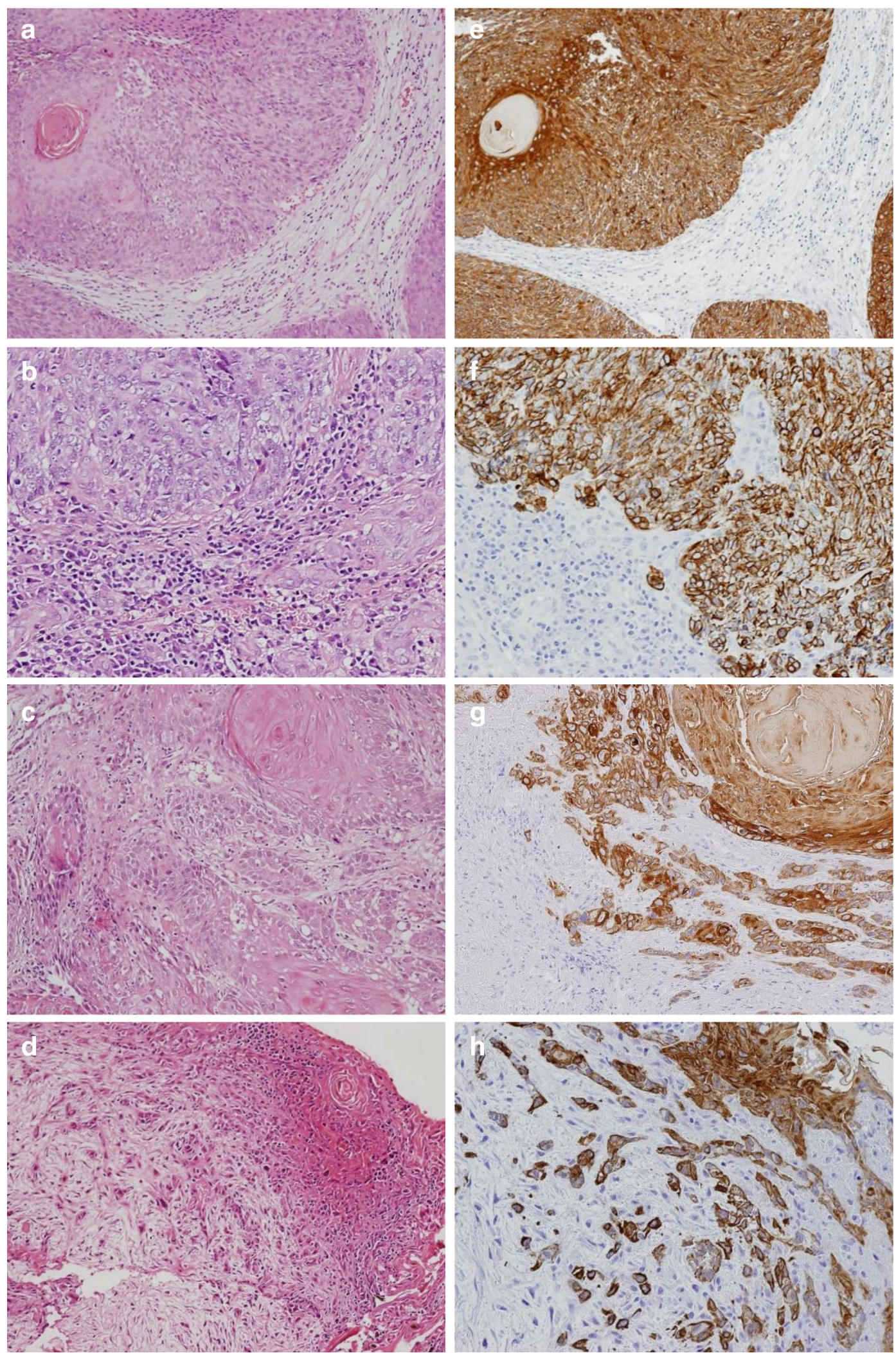

Fig. 1 Hematoxylin-eosin staining of SCC of the EAC showing tumor budding/sprouting (a-d) and cytokeratin immunohistochemistry (CK-IHC) for tumor budding/sprouting (e-h) at the invasive front. Inset in $\mathbf{a}$, e shows absence of tumor budding; budding grade 0 , the others shows presence of tumor budding; $\mathbf{b}, \mathbf{f}$ shows budding grade 1, $\mathbf{c}, \mathbf{g}$ shows budding grade 2 , and $\mathbf{d}, \mathbf{h}$ shows budding grade 3 

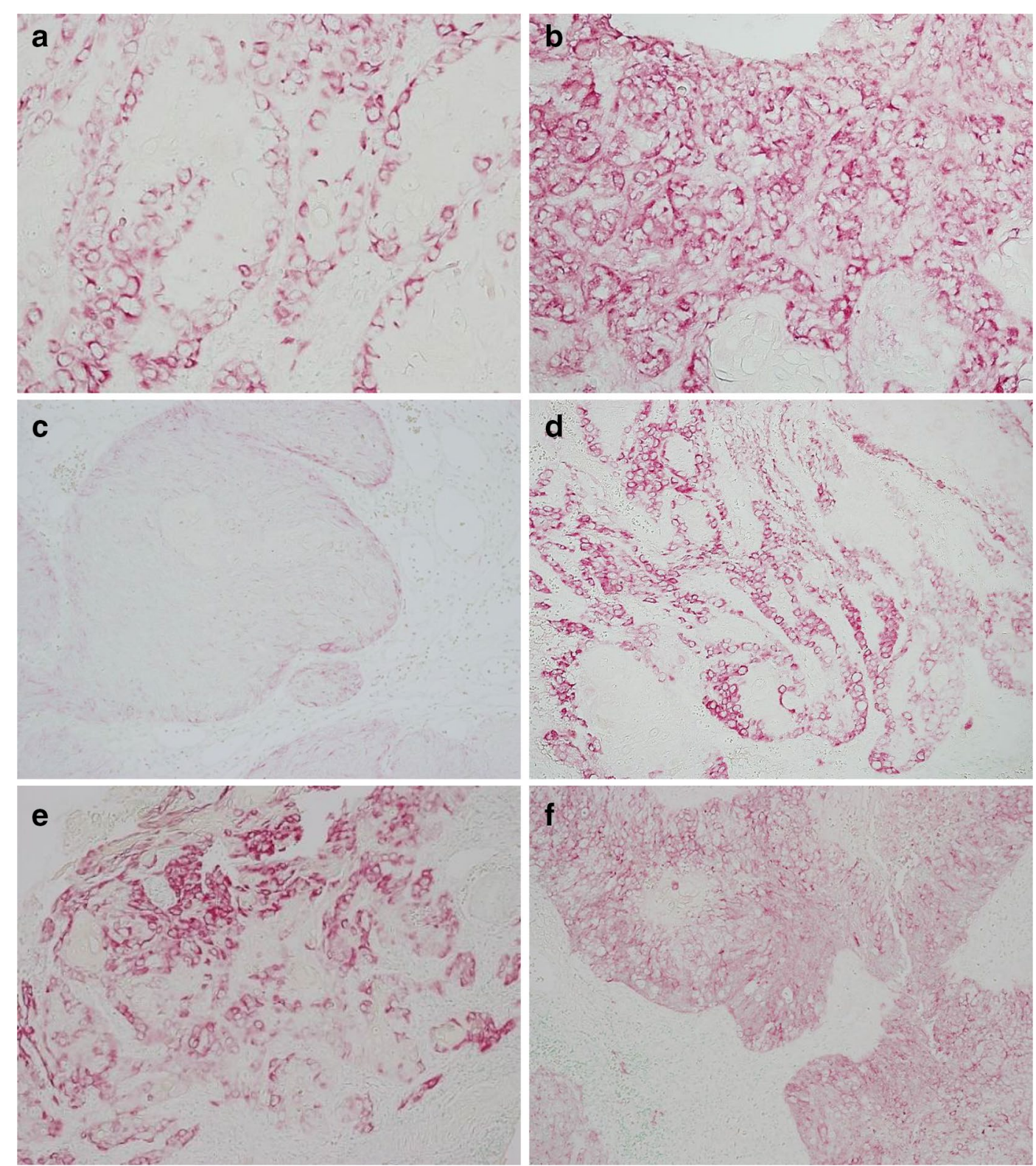

Fig. 2 Immunohistochemical analysis of Ln5- 2 2 expression in SCC of the EAC. a Marginal expression pattern. b Diffuse expression pattern. The expression level is scored as $1+$ to $4+$ based on the percentage of positively stained tumor cells. c $1+: 0-24 \%$, d 2+: 25-49 \%, e 3+: 50-74 \%, f 4+: $75-100 \%$

$38-86$ years $($ mean $=62.5)]$. The median follow-up period was 34.6 months (range 4-66 months). The TNM stages for a majority of tumors was stage IV [25 (54.3\%)]. The proportion of distant metastasis was low (4.3\%), similar to that reported in previous studies (Moffat et al. 2000; Moody et al. 2000; Nakagawa et al. 2006; Bacciu et al. 2013; Prasad et al. 2014; Masterson et al. 2014). The proportion of patients in which the cancer had spread to the lymph nodes was $21.7 \%$, slightly higher than that in previous studies (ranging from 10 to $15 \%$ ) (Moffat et al. 2000).
Because all the metastatic nodes were in an advanced stage according to the staging protocol proposed by University of Pittsburgh, we considered N1, N2a, and N2b as $\mathrm{N}+$. The presence of lymphovascular invasion could not be assessed because of the size of the biopsy specimens. Lateral temporal bone resection was achieved in 15 cases, subtotal temporal bone resection was achieved in 16 cases, and 15 cases received only chemo-radiotherapy. Of the 31 (69.6\%) patients who underwent surgery, postoperative data was available for $21(65.6 \%)$ patients and 
Table 1 Clinicopathological features of 46 patients with SCC of EAC and their correlation to tumor growth pattern and budding grade

\begin{tabular}{|c|c|c|c|c|c|c|c|}
\hline & \multirow{2}{*}{$\begin{array}{l}\text { All } \\
\text { n (\%) }\end{array}$} & \multicolumn{3}{|c|}{ Tumor budding } & \multicolumn{3}{|c|}{ Budding grade } \\
\hline & & $\begin{array}{l}\text { Absent } \\
13(28.3)\end{array}$ & $\begin{array}{l}\text { Present } \\
33(71.7)\end{array}$ & $P$ value & $\begin{array}{l}\text { Low } \\
39(84.8)\end{array}$ & $\begin{array}{l}\text { High } \\
7(15.2)\end{array}$ & $P$ value \\
\hline Mean age [years, $(\mathrm{sd})]$ & $62.5( \pm 11.4)$ & $65.0( \pm 3.1)$ & $61.5( \pm 1.9)$ & 0.358 & $63.1( \pm 1.8)$ & $59.3( \pm 0.4 .3)$ & 0.426 \\
\hline M:F & $21: 25(46: 54)$ & $8: 5(62: 38)$ & $17: 16(52: 48)$ & 0.743 & $21: 18(54: 46)$ & $4: 3(57: 43)$ & 1.000 \\
\hline \multicolumn{8}{|l|}{$\mathrm{T}$} \\
\hline $\mathrm{T} 1$ & $1(2.2)$ & & & & & & \\
\hline $\mathrm{T} 2$ & $12(26.1)$ & $4(30.8)$ & $9(27.3)$ & & $11(28.2)$ & $2(28.6)$ & \\
\hline T3 & $9(19.5)$ & & & & & & \\
\hline T4 & $24(52.2)$ & $9(69.2)$ & $24(72.7)$ & 1.000 & $28(84.8)$ & $5(71.4)$ & 1.000 \\
\hline \multicolumn{8}{|l|}{ N } \\
\hline No & $36(78.3)$ & $11(84.6)$ & $25(75.8)$ & & $31(79.5)$ & $5(71.4)$ & \\
\hline $\mathrm{N}+$ & $10(21.7)$ & $2(15.4)$ & $8(24.2)$ & 0.700 & $8(20.5)$ & $2(28.6)$ & 0.636 \\
\hline \multicolumn{8}{|l|}{ M } \\
\hline MO & $44(95.7)$ & $13(100)$ & $31(94.0)$ & & $38(97.4)$ & $6(85.7)$ & \\
\hline$M+$ & $2(4.3)$ & $0(0)$ & $2(6.0)$ & 1.000 & $1(2.6)$ & $1(14.3)$ & 0.284 \\
\hline \multicolumn{8}{|l|}{ TNM stage } \\
\hline Stage I & $1(2.2)$ & & & & & & \\
\hline Stage II & $10(21.7)$ & $4(30.8)$ & $7(21.2)$ & & $10(25.6)$ & $1(14.3)$ & \\
\hline Stage III & $10(21.7)$ & & & & & & \\
\hline Stage IV & $25(54.4)$ & $9(69.2)$ & $26(78.8)$ & 0.702 & $29(74.4)$ & $6(85.7)$ & 1.000 \\
\hline \multicolumn{8}{|l|}{ Differentiation } \\
\hline Well & $28(60.9)$ & $7(53.8)$ & $21(63.6)$ & & $26(66.7)$ & $2(28.6)$ & \\
\hline Moderate & $15(32.6)$ & $6(46.2)$ & $9(27.3)$ & & $11(28.2)$ & $4(57.1)$ & \\
\hline Poor & $3(6.5)$ & $0(0)$ & $3(9.1)$ & 0.309 & $2(5.1)$ & $1(14.3)$ & 0.157 \\
\hline Recurrence & 21 & & & & & & \\
\hline Free & 15 (71.4) & $4(80.0)$ & $11(68.8)$ & & 15 (78.9) & $0(0)$ & \\
\hline Recurrence & $6(28.6)$ & $1(20.0)$ & $5(31.2)$ & 1.000 & $4(21.1)$ & $2(100)$ & 0.071 \\
\hline Effect of therapy & 18 & & & & & & \\
\hline Grade 0-2 & $11(61.1)$ & $1(25.0)$ & $10(71.4)$ & & $9(60.0)$ & $2(66.7)$ & \\
\hline Grade 3 & $7(38.9)$ & $3(75.0)$ & $4(28.6)$ & 0.245 & $6(40.0)$ & $1(33.3)$ & 1.000 \\
\hline
\end{tabular}

$6(29 \%)$ patients had postoperative local recurrence. Of these 21 patients, $18(85.7 \%)$ had received neoadjuvant chemo-radiotherapy. Of these patients, we assessed the effect of therapy using surgical tissue specimens, according to the General Rules for Clinical Studies on Head and Neck Cancer (Cancer JSfHaN 2012). The effect of therapy was classified as one case (5.6\%) with a grade 0 , one $(5.6 \%)$ with a grade $1,9(50.0 \%)$ with a grade 2 , and 7 $(38.9 \%)$ with a grade 3 , response.

\section{Tumor budding in pre-treatment biopsy samples}

Tumor budding was first assessed in H\&E-sections (Fig. 1a-d). However, assessment was sometimes difficult because of the presence of reactive fibroblasts or macrophages in the stroma surrounding the invasive front. Therefore, we assessed budding carcinoma cells based on
CK-IHC data (Fig. 1e-h), according to a method previously reported by Satoh et al. (2014).

The results for the budding data grading indicated that $13(28.3 \%)$ patients had tumors graded as 0, 20 (43.5\%) patients had grade 1 tumors, $6(13.0 \%)$ patients had grade 2 tumors, and 7 (15.2\%) patients had grade 3 tumors. Thirty-nine $(84.8 \%)$ patients had low budding grade and 7 (15.2\%) patients had high budding grade tumors. There was no significant association between tumor budding and the clinicopathological parameters (Table 1).

Expression of $\operatorname{Ln} 5-\gamma 2$ and clinicopathological correlations Immunoreactivity for Ln5- $\gamma 2$ was observed in all SCC of the EAC cases in this study. All tumors demonstrated cytoplasmic and membranous staining. The expression level was as follows: 20 patients $(43.5 \%)$ as $1+, 17$ 
$(37.0 \%)$ as $2+, 6(13.0 \%)$ as $3+$, and $3(6.5 \%)$ as $4+$. Thirty-seven $(80.4 \%)$ patients had tumors with high expression of Ln5- $\gamma 2(1+, 2+)$. Nine (19.6\%) patients had low levels $(3+, 4+)$ of Ln5- $\gamma 2$ expression. The Ln5$\gamma 2$ expression patterns showed significant associations with age, advanced TNM stage (stage III and IV), and poor tumor differentiation $(P=0.0161, P=0.0439$, and $P=0.0200$, respectively). High Ln5- $\gamma 2$ expression levels were significantly associated with older age $(P=0.0224)$.

\section{Tumor budding and Ln5- $\gamma 2$ expression}

The results for the relationships between tumor budding and Ln5- $\gamma 2$ expression are summarized in Table 2. There was a significant correlation between presence of tumor budding and high Ln5- $\gamma 2$ expression levels $(P=0.0444)$. High budding grade correlated significantly with both a diffuse Ln5- $\gamma 2$ expression pattern and high Ln5- $\gamma 2$ expression levels $(P=0.0458$ and $P=0.0016$, respectively).

\section{Patients survival}

Disease-specific survival curves, sorted by the presence of tumor budding (Fig. 3a), budding grade (Fig. 3b), and the patterns (Fig. 3c) and levels (Fig. 3d) of Ln5- $\gamma 2$ expression were analyzed. The patient group with high tumor budding grades had significantly shorter survival times $(P=0.0007)$, but there was no statistically significant difference between the groups in terms of the presence of tumor budding $(P=0.4055)$. The patient groups with a diffuse Ln5- $\gamma 2$ expression pattern and high Ln5- $\gamma 2$ expression levels also had significantly shorter survival times ( $P=0.0337$ and $P=0.0079$, respectively).

We also analyzed disease-specific survival in cases with only advanced stages (stage III and IV) of SCC of the EAC, sorted into groups based on the budding grade (Fig. 4a) and Ln5- $\gamma 2$ expression levels (Fig. 4b). The patient groups with high budding grades and high Ln5- $\gamma 2$ expression levels had significantly shorter survival times
( $P=0.0027$ and $P=0.0484$, respectively), even when the analysis was restricted to only those cases with advanced stage disease.

Univariate and multivariate analyses of clinicopathological predictors of disease-specific survival in these cases of SCC of the EAC were also performed. The results indicated that advanced age, patients treated without surgery and high budding grades predicted poorer diseasespecific survival (Table 3 ). The high budding grade was an independent prognostic factor for poorer diseasespecific survival in patients with stage III and IV cancer $(P=0.010)$ (Table 3$)$.

\section{Discussion}

To the best of our knowledge, this study was the first to assess tumor budding/sprouting by grade in SCC of the EAC samples. We found that high budding grade was significantly correlated with poor prognosis in patients with SCC of the EAC. The results of a multivariate analysis indicated that high budding grade was an independent poor prognostic factor even in cases with advanced stage disease, although the number of patients analyzed was small particularly when considering the subgroups for statistical analysis (e.g. 7 patients with high budding grade). We also analyzed Ln5- $\gamma 2$ expression levels, which have been documented in the literature as being associated with tumor budding (Pyke et al. 1995; Sordat et al. 2000; Nabeshima et al. 2006). The Ln5- $\gamma 2$ expression level was significantly associated with tumor budding and budding grade. High Ln5- $\gamma 2$ expression was also significantly associated with shorter disease-specific survival.

Tumor budding/sprouting is a poor prognostic indicator in several types of SCCs (Koike et al. 2008; Zlobec and Lugli 2010; Brown et al. 2010). In the head and neck SCC, the tumor frequently shows higher grade of cellular dissociation at the invasive front than the remaining areas of the tumor, and grading of the invasive tumor front or margins is an independent prognostic factor in

Table 2 Association of tumor budding and Ln5- $\mathbf{2}$ expression

\begin{tabular}{|c|c|c|c|c|c|c|c|}
\hline & \multirow{2}{*}{$\begin{array}{l}\text { All } \\
\text { n (\%) }\end{array}$} & \multicolumn{2}{|c|}{ Tumor budding } & \multirow[t]{2}{*}{$P$ value } & \multicolumn{2}{|c|}{ Budding grade } & \multirow[t]{2}{*}{$P$ value } \\
\hline & & $\begin{array}{l}\text { Absence } \\
13(28.3)\end{array}$ & $\begin{array}{l}\text { Presence } \\
33(71.7)\end{array}$ & & $\begin{array}{l}\text { Low } \\
39(84.8)\end{array}$ & $\begin{array}{l}\text { High } \\
7(15.2)\end{array}$ & \\
\hline Ln5- $\gamma 2$ expression pattern & 46 & & & & & & \\
\hline Marginal & $35(76.1)$ & $10(76.9)$ & $25(75.9)$ & & $32(82.1)$ & $3(42.9)$ & \\
\hline Diffuse & $11(23.9)$ & $3(23.1)$ & $8(24.1)$ & 1.000 & $7(17.9)$ & $4(57.1)$ & 0.045 \\
\hline \multicolumn{8}{|l|}{ Ln5-ү2 expression level } \\
\hline Low & $37(80.4)$ & $13(100)$ & $24(72.7)$ & & $35(89.7)$ & $2(28.6)$ & \\
\hline High & $9(19.6)$ & $0(0)$ & $9(27.2)$ & 0.044 & $4(10.3)$ & $5(71.4)$ & 0.001 \\
\hline
\end{tabular}



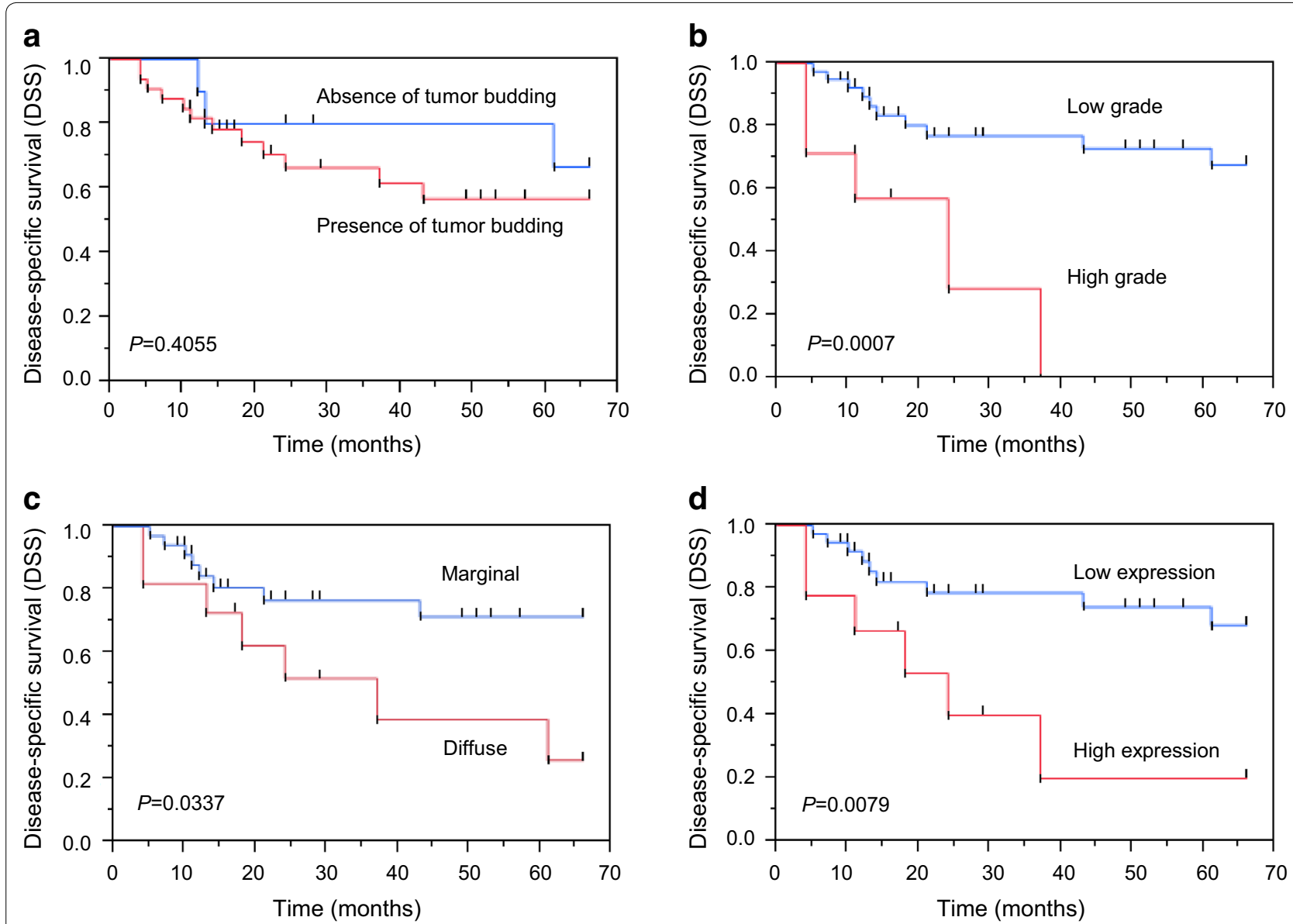

Fig. 3 Kaplan-Meier survival curve relating to absent or present of tumor budding (a), budding grade (b), Ln5- -2 expression pattern (c), and Ln5- $\gamma 2$ expression level (d) in the pre-treatment biopsy specimens from all cases in this study
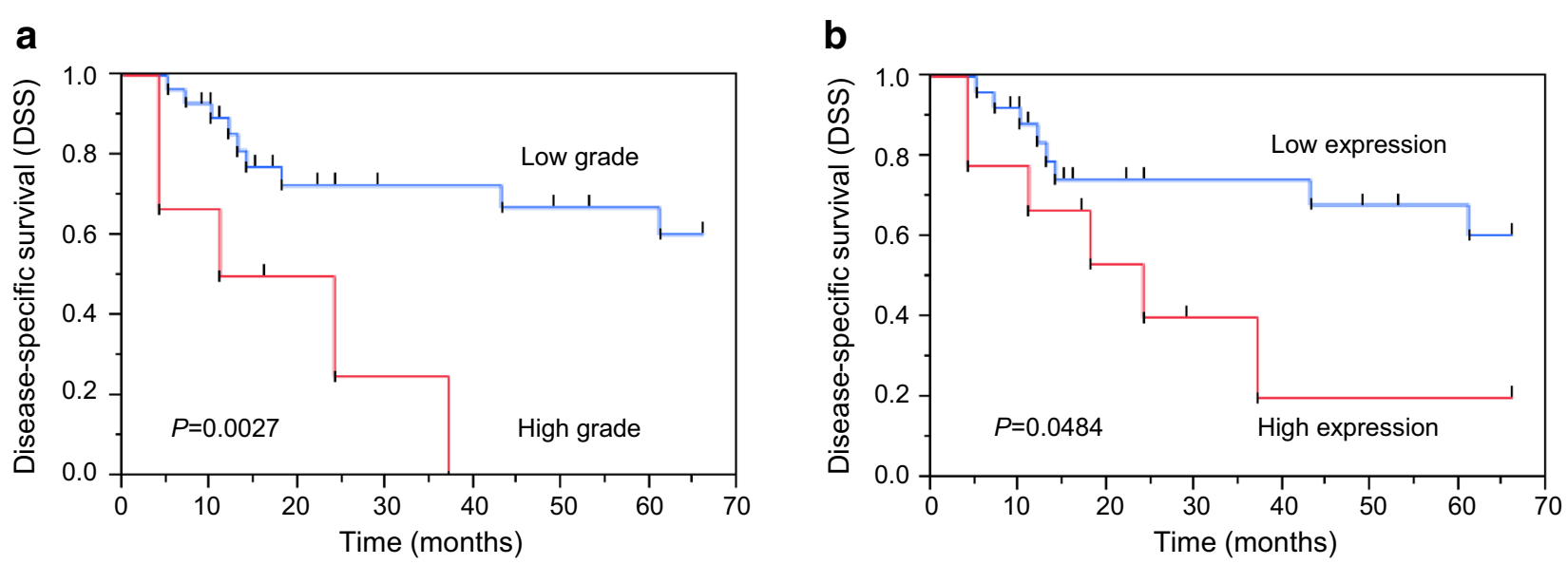

Fig. 4 Kaplan-Meier survival curve relating to budding grade (a) and Ln5- $\gamma 2$ expression level (b) in the pre-treatment biopsy specimens from only advanced stage (stage III and IV) cancer patients 
Table 3 Univariate and multivariate analyses of factors predisposing to disease-specific survival in only cases with stage III and IV cancer

\begin{tabular}{|c|c|c|c|c|c|c|}
\hline \multirow[t]{2}{*}{ Variable } & \multicolumn{3}{|c|}{ Univariate analysis } & \multicolumn{3}{|c|}{ Multivariate analysis } \\
\hline & Hazard ratio & $95 \% \mathrm{Cl}$ & $P$ value & Hazard ratio & $95 \% \mathrm{Cl}$ & $P$ value \\
\hline Age $\geq 70$ years & 3.163 & $1.038-9.130$ & 0.043 & 4.243 & $0.999-17.759$ & 0.050 \\
\hline Sex (male) & 1.247 & $0.432-3.796$ & 0.682 & 2.515 & $0.679-10.827$ & 0.168 \\
\hline Poor differentiation & 3.891 & $0.861-13.096$ & 0.073 & 1.843 & $0.284-10.948$ & 0.504 \\
\hline Patients treated without surgery & 3.710 & $1.251-11.643$ & 0.018 & 2.376 & $0.628-9.456$ & 0.199 \\
\hline High budding grade & 4.930 & $1.449-15.574$ & 0.012 & 11.914 & $1.772-96.778$ & 0.010 \\
\hline High Ln5- $\gamma 2$ expression level & 2.829 & $0.915-8.332$ & 0.069 & 0.797 & $0.114-4.486$ & 0.805 \\
\hline
\end{tabular}

multivariate survival analysis (Bryne et al. 1992, 1998). In our study, most of the patients with tumor budding had shorter disease-specific survival times. On the contrary, among the patients with early stage disease (stage I and II), those with no tumor budding survived until the end of the follow-up period (66 months). Several reports have described the relationships between clinicopathological parameters and tumor budding. Morodomi et al. (1989) reported that tumor budding may be a prelude to lymphatic invasion. Koike et al. (2008) reported that tumor budding correlates significantly with lymph node metastasis, lymphatic and vessel invasion, and intramural metastasis in SCC of the esophagus. Similarly in the auricular SCC, Clark et al. (2010) reported that a non-cohesive invasive front is an indicator of lymph node metastasis. However, in our study, regional lymph node status was not significantly associated with tumor budding. The small number and proportion $(21.7 \%)$ of cases with lymph node metastasis included in our study might have contributed to these differences.

Expression of Ln5- $\gamma 2$ in budding cells is associated with focal under-expression of the E-cadherin- $\beta$-catenin complex in colorectal carcinoma (Nabeshima et al. 2006). The presence of tumor budding is considered to represent the EMT, a process frequently associated with increased expression of molecules relevant to tumor invasion, such as matrix metalloproteinases and Ln5$\gamma 2$ in tumor cells, and stimulation of the Wnt signaling pathway (Zlobec and Lugli 2010). One study found that there is a link between Ln5- $\gamma 2$-mediated budding activity and decreased cell-cell adhesion (Nabeshima et al. 2006). Similarly, our study revealed that Ln5- $\gamma 2$ expression was significantly correlated with budding grade. The patient groups with high Ln5- $\gamma 2$ expression levels had a significantly shorter survival time, regardless of the disease stage. These results indicated that there was an association between higher Ln5- $\gamma 2$ expression and a poor prognosis.

\section{Conclusion}

In conclusion, tumor budding may be an adverse prognostic factor that can help stratify patients into more meaningful risk groups than TNM staging alone, and more importantly, has a potential to guide clinical decision-making. For example, low budding grade enabled us to sort the patient groups into those with better prognosis even in cases of advanced stage diseases. The treatment of patients with advanced-stage SCC of the EAC includes performing an extended en bloc temporal bone resection, which leaves the patients with a functional and a cosmetic deficit. Our results indicated that tumor budding could allow us to perform less invasive surgery for patients with low budding grade tumors. Additionally, tumor budding can be evaluated with relative ease during a routine pathological examination. Because of the strong correlation between Ln5- $\gamma 2$ expression and tumor budding, we can use immunohistochemical analysis of Ln5$\gamma 2$ as an alternative or concurrent parameter when tumor budding cannot be assessed in the biopsy specimen. (e.g., when the sample is too small or does not including the stroma). Although biological or clinical malignancy of tumors depends on not only invasiveness but also proliferative ability of tumor cells, multivariate analysis in this study showed the mode of invasiveness is more important for survival than tumor size, stage, and differentiation in SCC of the EAC. Further studies are now in practice in our laboratory to examine the molecular factors and mechanisms of tumor budding.

\section{Abbreviations}

CK: cytokeratin; CT: computed tomography; DFS: disease-free survival; EAC: external auditory canal; EMT: epithelial-to-mesenchymal transition; Ln5- $\gamma 2$ : laminin 5- 2 2; MCF: middle cranial fossa; MRI: magnetic resonance imaging; SCC: squamous cell carcinoma.

\section{Author's contribution}

YaO: project development, data collection, data analysis, manuscript preparation. MA: project development, data analysis, manuscript editing. MH: project development, data analysis, manuscript editing. KK: project development, data analysis. TS: project development, data collection, data analysis. HS: project 
development, data collection, manuscript editing. YoO: project development, data collection, supervision. TN: project development, data collection, data analysis, manuscript editing, supervision. KN: project development, data analysis, manuscript editing, supervision. All authors read and approved the final manuscript.

\section{Author details}

${ }^{1}$ Department of Pathology, Fukuoka University School of Medicine and Hospital, 7-45-1 Nanakuma, Jonan-ku, Fukuoka 814-0180, Japan. ${ }^{2}$ Department of Otorhinolaryngology, Fukuoka University School of Medicine and Hospital, Fukuoka, Japan. ${ }^{3}$ Department of Otorhinolaryngology, Hamanomachi Hospital, Fukuoka, Japan. ${ }^{4}$ Department of Otorhinolaryngology, Graduate School of Medical Sciences, Kyushu University, Fukuoka, Japan. ${ }^{5}$ Department of Anatomic Pathology, Graduate School of Medical Sciences, Kyushu University, Fukuoka, Japan.

\section{Acknowledgements}

The authors would like to thank Ms. M. Onitsuka for her excellent technical assistance with the immunohistochemistry and Mr. F. Kiyomi for his assistance with the statistical analysis. This work was supported in part by grants from the Grants-in-Aid for Scientific Research from the Ministry of Education, Culture, Sports, Science, and Technology of Japan and the Research Center for Advanced Molecular Medicine, Fukuoka University.

\section{Competing interests}

The authors declare that they have no competing interests.

\section{Novelty and impact}

This is the first study to evaluate the tumor budding in squamous cell carcinoma of the external auditory canal. Tumor budding grades may assist to stratify patients into the more accurate risk group than TNM staging alone, and potentially contribute to clinical decision-making.

\section{Ethics statement}

The study protocol was approved by the Institutional Review Board (The Ethics Committee) of Kyushu University (No.26-185) and Fukuoka University (No.12-7-13) Hospitals.

Received: 27 August 2015 Accepted: 15 December 2015 Published online: 24 December 2015

\section{References}

Anneroth G, Batsakis J, Luna M (1987) Review of the literature and a recommended system of malignancy grading in oral squamous cell carcinoma. Scand J Dent Res 95:229-249

Bacciu A, Clemente IA, Piccirillo E, Ferrari S, Sanna M (2013) Guidelines for treating temporal bone carcinoma based on long-term outcomes. Otol Neurotol 34:898-907. doi:10.1097/MAO.0b013e318281e0a9

Brown M, Sillah K, Griffiths EA, Swindell R, West CM, Page RD, Welch IM, Pritchard SA (2010) Tumour budding and a low host inflammatory response are associated with a poor prognosis in oesophageal and gastro-oesophageal junction cancers. Histopathology 56:893-899. doi:10.1111/j.1365-2559.2010.03559.x

Bryne M, Koppang HS, Lilleng R, Kjaerheim A (1992) Malignancy grading of the deep invasive margins of oral squamous cell carcinomas has high prognostic value. J Pathol 166:375-381

Bryne M, Boysen M, Alfsen CG, Abeler VM, Sudbo J, Nesland JM, Kristensen GB, Piffko J, Bankfalvi A (1998) The invasive front of carcinomas. The most important area for tumor prognosis? Anticancer Res 18:4757-4764

Cancer JSfHaN (2012) General rules for clinical studies on head and neck cancer, 5th edn. Kanehara, Tokyo

Clark RR, Soutar DS, Hunter KD (2010) A retrospective analysis of histological prognostic factors for the development of lymph node metastases from auricular squamous cell carcinoma. Histopathology 57:138-146

Hamasaki H, Koga K, Aoki M, Hamasaki M, Koshikawa N, Seiki M, Iwasaki H, Nakayama J, Nabeshima K (2011) Expression of laminin 5-gamma2 chain in cutaneous squamous cell carcinoma and its role in tumour invasion. $\mathrm{Br}$ J Cancer 105:824-832. doi:10.1038/bjc.2011.283
Hase K, Shatney C, Johnson D, Trollope M, Vierra M (1993) Prognostic value of tumor "budding" in patients with colorectal cancer. Dis Colon Rectum 36:627-635

Imai T (1960) Growth patterns in human carcinoma. Their classification and relation to prognosis. Obstet Gynecol 16:296-308

Koike M, Kodera Y, Itoh Y, Nakayama G, Fujiwara M, Hamajima N, Nakao A (2008) Multivariate analysis of the pathologic features of esophagea squamous cell cancer: tumor budding is a significant independent prognostic factor. Ann Surg Oncol 15:1977-1982. doi:10.1245/ s10434-008-9901-6

Koshikawa N, Minegishi T, Nabeshima K, Seiki M (2008) Development of a new tracking tool for the human monomeric laminin-gamma 2 chain in vitro and in vivo. Cancer Res 68:530-536. doi:10.1158/0008-5472.CAN-07-5269

Luo WR, Gao F, Li SY, Yao KT (2012) Tumour budding and the expression of cancer stem cell marker aldehyde dehydrogenase 1 in nasopharyngeal carcinoma. Histopathology 61:1072-1081. doi:10.1111/j.1365-2559.2012.04350.x

Marioni G, Nucci R, Marino F, Giacomelli L, Rugge M, Pareschi R, Martini A (2012) Neoangiogenesis in temporal bone carcinoma: the prognostic role of CD105. Otol Neurotol 33:843-848. doi:10.1097/ MAO.0b013e318254edc9

Marioni G, Zanoletti E, Stritoni P, Lionello M, Giacomelli L, Gianatti A, Cattaneo L, Blandamura S, Mazzoni A, Martini A (2013) Expression of the tumour-suppressor maspin in temporal bone carcinoma. Histopathology 63:242-249. doi:10.1111/his.12151

Masterson L, Rouhani M, Donnelly NP, Tysome JR, Patel P, Jefferies SJ, Roques T, Scrase C, Mannion R, Macfarlane R, Hardy D, Durrani A, Price R, Marker A, Axon P, Moffat DA (2014) Squamous cell carcinoma of the temporal bone: clinical outcomes from radical surgery and postoperative radiotherapy. Otol Neurotol 35:501-508. doi:10.1097/mao.0000000000000265

Mazzoni A, Danesi G, Zanoletti E (2014) Primary squamous cell carcinoma of the external auditory canal: surgical treatment and long-term outcomes. Acta Otorhinolaryngol Ital 34:129-137

Mitrovic B, Schaeffer DF, Riddell RH, Kirsch R (2012) Tumor budding in colorectal carcinoma: time to take notice. Mod Pathol 25:1315-1325. doi:10.1038/modpathol.2012.94

Moffat DA, Chiossone-kerdel JA, Da. Crus M (2000) Squamous cell carcinoma In: Jackler RK, W. DCL (eds) Tumors of the ear and temporal bone. Lippincott Williams \& Wilkins Philadelphia, pp. 67-83

Moody SA, Hirsch BE, Myers EN (2000) Squamous cell carcinoma of the external auditory canal: an evaluation of a staging system. Am J Otol 21:582-588

Morodomi T, Isomoto H, Shirouzu K, Kakegawa K, Irie K, Morimatsu M (1989) An index for estimating the probability of lymph node metastasis in rectal cancers. Lymph node metastasis and the histopathology of actively invasive regions of cancer. Cancer 63:539-543

Nabeshima K, Iwasaki H, Kikuchi M, Suzumiya J (2006) Cohort migration and IQGAP1. In: Muto T, Mochizuki H, Masaki T (eds) Tumor Budding in colorectal cancer: recent progress in colorectal cancer research. Nova Science, New York, pp 117-136

Nakagawa T, Kumamoto Y, Natori Y, Shiratsuchi H, Toh S, Kakazu Y, Shibata S, Nakashima T, Komune S (2006) Squamous cell carcinoma of the external auditory canal and middle ear: an operation combined with preoperative chemoradiotherapy and a free surgical margin. Otol Neurotol 27:242248. doi:10.1097/01.mao.0000190463.88873.3d (discussion 249)

Nakagawa T, Sueta T, Nabeshima K (2013) Surgical management of T1 and T2 lesion with outcomes. In: Takahashi H (ed) Cholesteatoma and ear surgery — an update. Kugler, Amsterdam, pp 133-136

Nguyen BP, Ryan MC, Gil SG, Carter WG (2000) Deposition of laminin 5 in epidermal wounds regulates integrin signaling and adhesion. Curr Opin Cell Biol 12:554-562

Prasad SC, D'Orazio F, Medina M, Bacciu A, Sanna M (2014) State of the art in temporal bone malignancies. Curr Opin Otolaryngol Head Neck Surg 22:154-165. doi:10.1097/moo.0000000000000035

Pyke C, Salo S, Ralfkiaer E, Romer J, Dano K, Tryggvason K (1995) Laminin-5 is a marker of invading cancer cells in some human carcinomas and is coexpressed with the receptor for urokinase plasminogen activator in budding cancer cells in colon adenocarcinomas. Cancer Res 55:4132-4139

Rectum JSfCotCa (2013) Japanese classification of colorectal carcinoma, 8th edn. Kanehara, Tokyo 
Rogers AC, Gibbons D, Hanly AM, Hyland JM, O'Connell PR, Winter DC, Sheahan K (2014) Prognostic significance of tumor budding in rectal cancer biopsies before neoadjuvant therapy. Mod Pathol 27:156-162. doi:10.1038/modpathol.2013.124

Sarioglu S, Acara C, Akman FC, Dag N, Ecevit C, Ikiz AO, Cetinayak OH, Ada E, for Dokuz Eylul H, Neck Tumour G (2010) Tumor budding as a prognostic marker in laryngeal carcinoma. Pathol Res Pract 206:88-92. doi:10.1016/j. prp.2009.09.006

Satoh K, Nimura S, Aoki M, Hamasaki M, Koga K, Iwasaki H, Yamashita Y, Kataoka H, Nabeshima K (2014) Tumor budding in colorectal carcinoma assessed by cytokeratin immunostaining and budding areas: possible involvement of c-Met. Cancer Sci 105:1487-1495. doi:10.1111/cas.12530

Sordat I, Rousselle P, Chaubert P, Petermann O, Aberdam D, Bosman FT, Sordat B (2000) Tumor cell budding and laminin-5 expression in colorectal carcinoma can be modulated by the tissue micro-environment. Int $J$ Cancer 88:708-717

Ueno H, Murphy J, Jass JR, Mochizuki H, Talbot IC (2002) Tumour 'budding' as an index to estimate the potential of aggressiveness in rectal cancer. Histopathology 40:127-132
Willen R, Nathanson A, Moberger G, Anneroth G (1975) Squamous cell carcinoma of the gingiva. Histological classification and grading of malignancy. Acta Oto-laryngol 79:146-154

Yamamoto H, Itoh F, Iku S, Hosokawa M, Imai K (2001) Expression of the gamma(2) chain of laminin-5 at the invasive front is associated with recurrence and poor prognosis in human esophageal squamous cell carcinoma. Clin Cancer Res 7:896-900

Zlobec I, Lugli A (2010) Epithelial mesenchymal transition and tumor budding in aggressive colorectal cancer: tumor budding as oncotarget. Oncotarget 1:651-661

\section{Submit your manuscript to a SpringerOpen ${ }^{\odot}$ journal and benefit from:}

- Convenient online submission

- Rigorous peer review

- Immediate publication on acceptance

- Open access: articles freely available online

- High visibility within the field

- Retaining the copyright to your article

Submit your next manuscript at $>$ springeropen.com 